\title{
The Be Phenomenon: Connections to B-supergiants
}

\author{
Andreas Kaufer \\ European Southern Observatory, \\ Alonso de Cordova 3107, Santiago 19, Chile
}

\begin{abstract}
In this contribution the spectral characteristics, physical properties, and the evolutionary status of B-type supergiants is reviewed with respect to the Be phenomenon. Long-term monitoring campaigns in the UV and the optical have recently revealed the distinct variability patterns of the photospheric and the emission-line spectra of this class. The various proposed scenarios for the spatially structured circumstellar envelopes and their rotational modulation are presented. The possibility of generating and maintaining large-scale wind structures by photospheric processes which structure the underlying stellar surfaces is discussed. Some open questions in connection with these hypotheses will be identified.
\end{abstract}

\section{Introduction}

Early-type supergiants are well-separated from classical Be-stars by their evolutionary status and location in the HR Diagram. The OBA supergiants are the intrinsically brightest stars in the optical range and populate the uppermost regions of the HRD with luminosities of $>10^{5} \mathrm{~L}_{\odot}$. Stellar evolution models derive masses of $>20 \mathrm{M}_{\odot}$ and hydrogen-burning stellar cores, i.e., they tell us that hot supergiants are evolved massive stars - well distinct from the near main sequence Be stars.

The emission-line spectra of moderately luminous OBA Ia supergiants display line emission almost exclusively in the $\mathrm{H} \alpha$ line with a large variety of line profiles smoothly going through all profile types of the classical Beals scheme. With increasing luminosity, emission becomes also visible in the higher Balmer and other wind sensitive lines and the P Cygni-type line profile usually dominates the spectrum as expected for a mass losing star with an extended and expanding stellar envelope.

The stationary spectra of hot supergiants in the spectral range from early $\mathrm{O}$ to late $\mathrm{A}$ and over the large range of observed luminosities are described in great detail by the radiation-driven wind theory (e.g. Kudritizki 1999 and references therein). In contrast to the classical Be stars, in the case of the hot supergiants radiation force on spectral lines is sufficient to drive a stellar mass loss, i.e, to drive and maintain a continuously expanding envelope around these stars.

Since we have such a clear distinction between the classes of Be stars and $\mathrm{B}$ supergiants, the hypothesis of this paper is that the link between the Be phenomenon and the hot supergiants - if any - has to be searched for by comparing 
the characteristics of their variability. From this, possibly the physical mechanisms in the photospheres and envelopes which are causing the variability could be identified.

Ground- and space-based long-term monitoring campaigns have resulted in the collection of increasing evidence for cyclic variability in the stellar winds of hot luminous stars. In most cases this variability is interpreted as rotational modulation of the stellar winds by either transient or even - on the time scale of several rotational cycles - persistent large-scale wind structures. Due to the large uncertainties in the estimates for the true rotation period of the underlying star, it is possible only in few cases to identify unambiguously the modulation period, i.e., the typical wind variation cycle time, with the stellar rotation period. Therefore, the identification of rotational modulation relies on time scale arguments as the cyclical component of the wind modulation is observed to be (i) considerably longer than the flow times of the wind and (ii) longer than the estimated pulsation time scales of the stellar photospheres.

In the following, the observational evidence for spatially structured stellar photospheres and spatially structured circumstellar envelopes in hot supergiants will be reviewed on the basis of case studies. Since it is widely believed that the large scale wind structures are rooted to the photospheric processes of the underlying star, the question for the various possibilities to generate such wind structures from structured stellar surfaces is discussed.

\section{Evidence for spatially structured stellar photospheres}

Photometric microvariability of early-type supergiants has first been systematically studied in the early 1970's by Sterken (1976) and Burki (1977). Up to now, long data sets, which for individual objects extend over more than 25 years have been accumulated with the help of e.g. the Long-Term Photometry of Variables (LTPV) program as initiated by Sterken (1983), Walraven photometry at the Dutch 90-cm telescope (van Genderen 1992), and recent photometry from the Hipparcos satellite (e.g. van Leeuwen et al. 1998 and references therein). Sterken et al. (1999) present a unique extended data set for the two late-type B supergiants HD 168607 (B9 Ia) and HD 168625 (B8 Ia).

The common findings of photometric studies of early supergiants are (i) amplitudes of up to a few tenths of a magnitude in the visible with little color variations and (ii) that the extended data sets can be described by multiperiodic sinusoidal signals. The derived periods can usually be identified with the dominant periods in the different observing seasons. The different periods are found to be non-identical from season to season but of the same order which permits the attribution of so-called "semi-periods" to individual objects. The derived semi-periods are in general identified with pulsation periods and cover a typical range of $10-100$ days. The values are generally longer than the expected radialfundamental pulsation periods of the stars. Therefore, the photometric variability of early-type supergiants has almost exclusively been identified - based on this time-scale argument - with non-radial, low-order, $g$-mode pulsations.

In the framework of the HEROS spectroscopic long-term monitoring project (cf. Kaufer 1998), a sample of six BA supergiants has been observed intensively over three observing seasons with high spectral resolution and a typical sampling 
of one spectrum per night. Kaufer et al. (1997) have performed a multi-line study of the photospheric radial-velocity variability in this data set. They conclude, in agreement with the photometric studies, that the photospheric periods of BA supergiants are confined between the maximal acceptable radial fundamental pulsation periods and the shortest possible rotation periods, i.e., the $g$-mode domain of non-radial pulsations (NRPs) (cf. also Lamers et al. 1998).

An 8-year LTPV photometric record and a spectroscopic time series from HEROS are available only for one program star, namely HD 92207 (A0 Ia), Unfortunately the two data sets do not overlap in time. However, the time series analysis of the Stroemgren $y$ lightcurve results in CLEANed periods with $3 \sigma$ significance of $148,62,44,27$, and 21 days. While the longest period is of the order of the expected rotation period of the star, the four shorter periods are in good agreement with the four most significant periods as found in the ClEANed two-dimensional (velocity - frequency) power spectra (Kaufer et al. 1997, their Fig. 10).

With this finding we have good evidence that the same physical mechanism in the stellar photosphere is responsible for the photometric variations and the photospheric line-profile variations (lpv's). On the other hand, HD 92207 is the only star in the Heros supergiant sample that showed a recurrent period (the 27-days period) over the three seasons with line-profile variations reminiscent of low-order prograde NRP patterns. All other supergiants show season-to-season variations of their spectroscopic (and photometric) period spectra. This is the reason that the identification of the variability with NRP $g$-modes remains a purely statistical one (Baade 1999).

A similar picture arises for the O-type supergiants where only $\zeta$ Pup (O4 $\mathrm{I}(\mathrm{n}) \mathrm{f}$ ) has been found to have a persistent period of $8.54 \mathrm{hrs}$ (Baade 1991, confirmed by Reid \& Howarth 1996) while for others like $\lambda$ Cep (O6 I(n)fp) (Henrichs 1991), HD 34656 (O7 II(f)) (Fullerton et al. 1991), $\xi$ Per (O7.5 III(n)((f))) (Gies \& Bolton 1986), and $\alpha$ Cam (O9.5İa) (Ebbets 1982) with lpv's have indicated possible NRP periods, but these have not yet been confirmed by independent data sets.

\section{Evidence for spatially structured circumstellar envelopes}

\subsection{Radial structures and their time evolution}

Rivinius et al. (1997) discussed HERos time series of very luminous early-type $\mathrm{B}$ hypergiants, like the proto-typical $\zeta^{1} \mathrm{Sco}\left(\mathrm{B} 1.5 \mathrm{Ia}^{+}\right)$. Numerous P-Cygni type lines in the optical spectra document a strong outward accelerating stellar wind with mass-loss rates of the order of $10^{-6} \mathrm{M}_{\odot} \mathrm{yr}^{-1}$. Bluewards accelerating discrete absorption components (DACs) in the dynamical spectra indicate disturbances propagating with the ambient stellar wind. For the first time in these objects the DACs could be traced from photospheric velocities up to close the terminal velocity of the wind. The derived "velocity-law" of the DACs shows an almost constant acceleration over a large velocity range, which, if parameterized by a $\beta$-type velocity law, would lead to $\beta$ parameters of some 2.5 whereas for the more rapidly accelerating, spherically symmetric, ambient wind a $\beta=1.5$ was derived by fitting stellar-wind models to the Balmer lines. The authors iden- 
tify the DACs with patches of material of enhanced density with respect to the ambient gas propagating radially outwards in the line of sight of the observer.

The quasi-photospheric lines in extreme objects like $\zeta^{1}$ Sco are not formed in hydrostatic equilibrium. Rather, the onset of the stellar wind is seen in a continuously increasing blue shift of the line-forming regions. Since the B hypergiants display qualitatively the same pulsation-like radial-velocity variations as the less extreme supergiants discussed in the previous section, $\zeta^{1}$ Sco gives the unique possibility to study the propagation of deep-seated photospheric pulsation disturbances into the stellar wind. Rivinius et al. (1997) discuss the increasing time delay of the observed pulsation patterns with increasing height of formation above the stellar surface. Further, the pulsation semi-periods and the recurrence cycle times of the DACs in the wind are of the same order, three weeks. Despite these encouraging observational hints for a photosphere-wind connection, no direct link between an outwards propagating pulsation pattern with a rising and developing DAC could be established.

\subsection{Azimuthal structures and their rotational modulation}

$\theta^{1}$ OriC (= HD 37022) is the visually brightest and hottest star of the Trapezium in the Orion nebula (M 42). It is a very young main sequence $O$ star of spectral type $\mathrm{O} 7 \mathrm{~V}$, i.e., not a supergiant, but is included here as a textbook example for an object with a rotationally modulated stellar wind. $\theta^{1}$ Ori $\mathrm{C}$ has been known for a long time for its spectroscopic peculiarities as described by Conti (1972) and Walborn (1981).

Stahl et al. (1993) discovered a strictly periodic 15.4-day modulation of the $\mathrm{H} \alpha$ and HeII $\lambda 4686$ emission lines of $\theta^{1}$ Ori C . The finding of this period then allowed to phase the CIV and SirV UV wind lines in IUE archival data (Walborn \& Nichols, 1994). With the help of simultaneous optical Heros and IUE UV spectra, Stahl et al. (1996) determined a precise period of $15.422 \pm 0.002$ days for the modulation of the optical and UV wind lines as well as for the photospheric lines.

The UV wind lines show an excess absorption almost instantly over the full velocity range of the stellar wind while the optical wind emission lines are weakest, whereas the maximum strength of the photospheric absorption lines falls together with the maximum emission of the optical wind lines.

Based on these phase relations Stahl et al. (1996) developed a sketch for $\theta^{1}$ Ori $\mathrm{C}$ with the stellar rotation as the driving clock of the modulations and a stellar envelope forced into corotation by a magnetosphere. Assuming an oblique magnetic rotator, the excess absorption in the UV wind lines originates above the magnetic pole while the excess emission of the optical wind lines is produced in an extended region of enhanced density near the magnetic equator, which has to coincide with a region of a magnetically disturbed photosphere to account for the variations of the photospheric lines.

Based on the 15.422-day period, pointed Rosat observations were carried out by Gagné et al. (1997) who found that the X-ray emission varies also in phase with the optical emission lines. Babel and Montemerle (1997) could quantitatively explain the X-ray flux and its variations with a "magnetically confined wind shock" model which gives further strong support for a magnetic origin of the variability. 
Therefore, $\theta^{1}$ Ori C seems to be closely related to the cooler He-variable magnetic stars but is so far unique among the O-type stars.

$H D 64760$ is an intrinsically fast rotating early-type $\mathrm{B}$ supergiant $(\mathrm{B} 0.5 \mathrm{Ib})$. From a 5.8-day time series of IUE spectra in 1993 it has been known to show cyclic variations in its UV "wind lines" (Massa et al. 1995a) and was selected as one of the three intensively monitored objects of the first IUE MEGA Campaign (Massa et al. 1995b).

Prinja et al. (1995) found a clearly periodic 1.2-day modulation in this continuous 15.8-day MEGA I time series. The dynamic spectra display a twocomponent variation: (i) a fast periodic modulation of the UV wind absorptions from nearly photospheric up to terminal wind velocities and (ii) a slowly evolving discrete absorption component (DAC) asymptotically approaching the terminal velocity over the length of the time series. Interestingly no link between the two types of variability could be established to date. This is a quite unexpected finding since wind variability due to DACs is the most commonly observed type of variability in $\mathrm{O}$ star winds and has been connected to the stellar rotation by different authors (cf. e.g. Prinja 1988; Kaper et al. 1996). Since rotational modulation is also the favored model for the newly discovered wind modulations in HD 64760 (see below) the conclusion must be that each of the two types of wind variation is caused by a different type of wind structure.

Fullerton et al. (1997) derive 1.202 \pm 0.004 and 2.44 \pm 0.04 -day periods for the fast sinusoidal modulation of the SiII, Sirv, and NV resonance lines of HD 64760 . The 2.4-day period is visible in the 1993 IUE data set and in the emission lobe of the UV P-Cygni profiles. In addition, a 2.40-day period is found with marginal significance in the $\mathrm{H} \alpha$-line of a HEROS times series with 97 optical spectra obtained in 1996 at the ESO 0.5-m telescope (cf. Fig. 1). The power distribution over the line shows two maxima at -250 and $+160 \mathrm{~km} \mathrm{~s}^{-1}$ and a local minimum at zero velocity (assuming a system velocity of $+18 \mathrm{~km} \mathrm{~s}^{-1}$ ). Therefore, the power distribution shows the same blueward asymmetry as the two emission peaks of the $\mathrm{H} \alpha$-profile which are located at -350 and $+260 \mathrm{~km} \mathrm{~s}^{-1}$. Howarth et al. (1998) could detect the 1.2-day period in the UV photospheric lines of the MEGA I data set with a weak signature for a prograde traveling NRP pattern in the corresponding phase diagrams. In the 1993 IUE data set, in parallel to the wind lines, the 2.4-day period is weakly present in the photospheric lines and slightly dominates the 1.2-day period.

Inspired by the fact that the detected wind-modulation periods are approximately a half and a quarter of the estimated rotational period of $P_{\text {rot }}=4.8$ days, Fullerton et al. (1997) proposed a broad two- and four-armed coratating circumstellar structure to modulate the optical depth of the stellar wind of HD 64760 . Owocki et al. (1995) developed a simple kinematic model to show that the "phase bowing" phenomenon observed by Fullerton et al., i.e., the simultaneous evolution of the modulations toward higher and lower velocities, is naturally explained by the projection of the velocity fields of corotating streak lines against the stellar disk. In fact phase bowing turns out to be a powerful tool to constrain the geometry of the wind-modulating structures. Cranmer \& Owocki (1996) further developed the idea of corotating streak lines into a consistent hydrodynamic model of corotating interaction regions (CIRs) caused by the collision of fast and slow wind streams. The wind streams are rooted in large-scale per- 

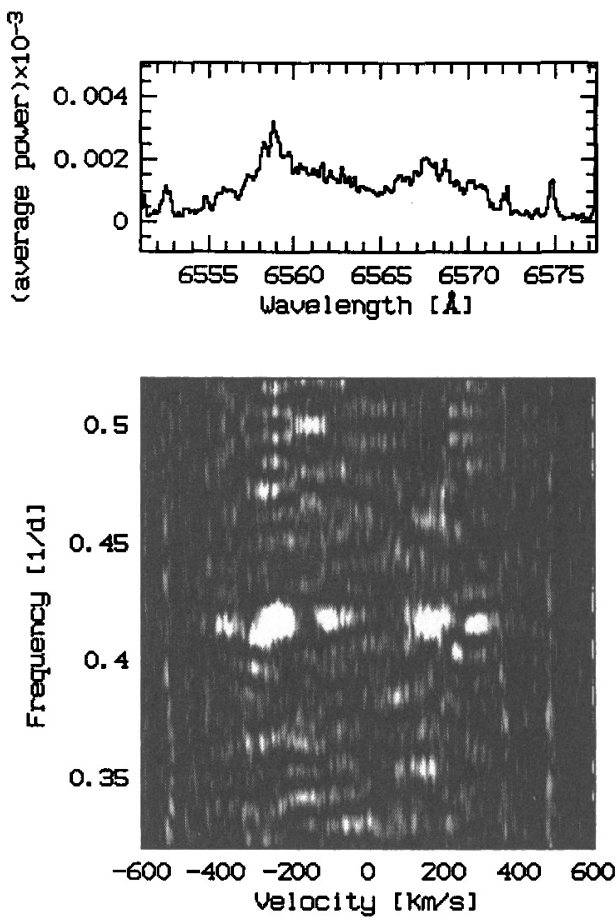

Figure 1. Two-dimensional power distribution over the $\mathrm{H} \alpha$ profile of HD 64760 in the frequency range near the 2.4-day period. The data set consisting of 97 Heros spectra with a typical $S / N \approx 150$ for the individual spectrum has been searched for statistically significant periods in the range of $0.1-1.0$ days $^{-1}$. The maximum power (with a $99 \%$ probability, only marginally significant) is found in the $\mathrm{H} \alpha$-line with the shown frequencies around 0.417 days $^{-1}$. The graph on the top shows the average power over the displayed frequency range as function of wavelength. Velocities are corrected for the system; see text.

turbations at the base of the wind. In the case of HD64760 a regular and longitudinally-spaced two- and four-sector geometry on the stellar surface seems quite applicable.

The 1.2-day NRP pattern, found by Howarth et al. (1998) in the photospheric UV absorption lines is a quite natural cause of such a regular geometry on the stellar surface. Unfortunately, the considerable uncertainties in the estimate for the true rotation period of the star, as discussed by the same authors, leave room for a fixed and strictly corotating surface pattern, as generated by possible magnetic surface structures or a prograde traveling wave pattern.

With the presence of the 1.2-day period in the photospheric lines, and the 2.4-day wind period in the P-Cygni emission lobes of the UV lines and in the $\mathrm{H} \alpha$ line at near-photospheric velocities, HD 64760 provides us with the best evidence to date for a photosphere-wind connection in hot supergiants. 
Late-type $B$ and early-type $A$ supergiants Kaufer et al. (1996a) presented and analyzed the complex variability patterns of the wind sensitive $\mathrm{H} \alpha$ line in the HEROS sample of BA supergiants as introduced above. The complex variability patterns in the examined BA supergiants turns out to be localized symmetrically about the system velocity with the maximum power of the variations just beyond the $\pm v \sin i$ photospheric velocities of the star - similar to the finding for HD 64760 in Fig. 1. The variations are mainly due to additional violet $(V)$ and red $(R)$ shifted emission components superimposed on the otherwise constant underlying wind or photospheric profiles. This characteristic $V$-and- $R$ variability was interpreted as indication for deviations of the circumstellar envelopes of the BA supergiants from spherical symmetry. Further - in analogy with Be stars - the double-peaked emission variability suggests an equatorial concentration of the emitting circumstellar material.

The equivalent-width curves of the variable "excess" emission show the cyclical character of the variability most clearly. The time-series analyses of the equivalent-width curves revealed one dominant period close to the rotational period as estimated from the $v \sin i$. Therefore, rotational modulation of the lower circumstellar envelope by stellar surface structures was proposed as the major source of the observed close-to-the-star wind variability. Weak magnetic surface structures could provide an efficient coupling of the stellar rotation into the lower wind region.

Further insight into the circumstellar structures in BA supergiants was obtained by the occasional observations of so-called "high-velocity absorptions" $(h v a)$ which were for the first time identified and described in detail by Kaufer et al. (1996b). The two most prominent and so far best documented hva's were observed in the dynamical $\mathrm{H} \alpha$ spectra of $\beta$ Ori (B8 Ia) in 1994 and HD 96919 (B9 Ia) in 1995. The $h v a$ 's are described as suddenly appearing, extraordinarily deep, and highly blue- but also beyond $+v \sin i$ red-shifted absorptions in the circumstellar envelope. The appearance of the hva's is announced either by $V$ or $V$-and- $R$ emission peaks in the "normal" $\mathrm{H} \alpha$ emission profile. The sudden appearance of the blue shifted absorptions within a few days in the velocity range $v_{\text {sys }} \rightarrow v_{\infty}$ is not compatible with the typical wind-flow times. Therefore, the hva's could not be explained as discrete mass-loss events at the base of the wind with a subsequent $\beta$-type acceleration of the "blob" within the ambient wind as described above for $\zeta^{1}$ Sco. In addition, the extreme depth of the absorptions suggests that the hva's are caused by extended regions in the envelope which consist of dense and therefore probably recombined circumstellar structures.

As for the case of HD 64760, a simple model of a rotating streak line with a rotating region of enhanced mass loss localized at the base of the wind near the stellar equator was proposed. This purely kinematic model primarily accounts for the sudden appearance of an $h v a$ because the streak line rotates into the line of sight of the observer. The complete velocity field $v_{\mathrm{sys}} \rightarrow v_{\infty}$ becomes visible as extended and also deep absorption against the stellar disk (since the observer looks along a large column of enhanced density). Assuming that the large streak line structures are stable for at least a few stellar rotation cycles, this model predicts the reappearance of the hva events after integer multiples of the stellar rotation period. For the case of the extreme hva event observed in HD 96919 in 1995, the reappearance dates of the maximum blue absorption 
were predicted with the estimated stellar rotation period of 93 days and an $h v a$ event with a strikingly similar time-velocity structure was observed by Kaufer et al. during a 1996 HeRos run in La Silla - exactly after four rotational cycles.

Israelian et al. (1997) reported on the observation of an hva event in $\beta$ Ori in 1993. Their time series loosely covers the evolution of the hva event in $\mathrm{H} \alpha$ from a strong and highly blueshifted absorption into a strong and highly redshifted absorption within a period of 30 days (cf. their Figs. 4 and 9). The two spectra with the maximum blue and red absorption are accompanied by red and blue emission, respectively. The authors proposed a rotating magnetic loop in the equatorial plane to explain the velocity and time signature of their spectra. In analogy to cool solar loops, the material flows along the upward leg and reaches the maximum velocity between the foot point and the highest point. The downward flow along the loop is slower and reaches its minimum velocity at the foot point of the downward leg. If the magnetically confined upward flow rotates into the line of sight of the observer and the flow projects against the stellar disk, a blueshifted absorption becomes visible. At the same time, the downward flow stays just off the stellar disk, i.e., is not projected against the stellar disk and therefore accounts for the simultaneous red emission. After about one quarter of the rotation period (assuming a 90 degree separation in longitude of the foot points, i.e., after $\Delta t=30$ days) the picture reverses. Then the red absorption is seen against the disk as a downward flow, together with blue emission from the upward stream in the loop.

The proposed rotating loop model accounts well for the observed spectral features of this $h v a$ in $\beta$ Ori - especially the deep redshifted absorption with a maximum velocity of $+130 \mathrm{~km} \mathrm{~s}^{-1}$, which is clearly beyond $+v \sin i \approx 50 \mathrm{~km} \mathrm{~s}^{-1}$. Otherwise, this velocity is difficult to explain by any other scenario. Interestingly, a dense HEROs time series obtained four months (= one rotation period) later, clearly displays the bona fide same $h v a$ with a strong blue absorption but without any sign for the corresponding red absorption 30 days later (cf. Kaufer et al. 1996b, their Fig. 2). In the loop model this might be interpreted as the closed magnetic loop having changed into an open loop configuration.

\section{Generation of large scale wind structures from structured stellar surfaces}

\subsection{Summary}

From the case studies of hot supergiants presented above, the following scheme for the different types of observed wind variability and their links to photospheric processes can be derived:

- Strict periodicity of wind modulation and coherence over long timescales favors stellar rotation as the driving "clock" and large scale magnetic fields to structure the stellar envelope (cf. $\theta^{1}$ Ori C).

- Periodic wind modulation with periods of integer fractions of stellar rotation periods favors rotation of equally spaced longitudinal sectors on the stellar surface as most naturally created by NRP patterns (cf. HD 64760). 
- Cyclic (i.e., not strictly periodic, not long-term coherent) wind modulation can only suggest rotational modulation via distributed surface structures. Whether these structures are created by local magnetic fields or complex multi-periodic pulsation patterns remains an open question, as in the case of late B-type supergiants like $\beta$ Ori and HD 92207.

- Transient large scale wind structures (as observed in DACs and hva's) point to individual active regions on the stellar surface which again can have either a magnetic ( $\beta$ Ori , HD 96919) or a dynamic $\left(\zeta^{1} \mathrm{Sco}\right)$ origin.

\subsection{Critical points}

The above scheme can be confronted immediately with the objection: has it been has generalized from too few prototypical cases? This is definitely true, and a quite natural result of strong selection effects. The extraordinarily high expense of observing time required to obtain this type of spectroscopic and photometric times-series data emphasize this point. It is quite indicative that most windvariability studies concentrated on the extreme objects, (in the sense of mass loss and luminosity) before a real "breakthrough" in this field came with a less luminous, normal B supergiant like HD 64760.

Unfortunately, the further interpretation of the large amount of collected time-series data suffers from the uncertainties of the fundamental stellar parameters. The discussion about rotational modulation is in almost all cases based only on rough estimates of the stellar rotation period $P_{\text {rot }} / \sin i$. Precise measurements of stellar radii are badly needed and will be hopefully obtained in the future with facilities like the VLTI. Even the precise measurements of an apparently simple quantity like $v \sin i$ requires in the case of early-type supergiants a deeper understanding of additional line-broadening mechanisms acting in their atmospheres. Up to now, the width of the variable part of the absorption-line profile seems a better but more indirect measure than the width of line profile itself (Reid et al. 1993).

In the case of early-type supergiants the step is a large one from the finding of signatures of radial and nonradial stellar pulsation towards the goal of doing real asteroseismology, and it is further hampered by the generally strong variability of the period spectra themselves. The recent advances in the detailed modeling of stellar pulsation and rotation (Townsend 1997) allow us to exploit maximally the information carried in the photospheric line-profile variations of the recorded spectroscopic time series.

On the other hand, magnetic field measurements with a precision of $1-$ $10 \mathrm{G}$ are needed to eventually identify stellar surface structures of magnetic origin. Simple estimates show that weak global fields of this order of magnitude are sufficient to produce large scale wind structures in the winds of early-type supergiant stars (cf. e.g. Mihalas \& Conti 1980). In this respect it is quite disappointing that the detection of a magnetic field in $\theta^{1}$ Ori $C$ as a primary candidate is still outstanding. 


\section{The role of Be stars}

Be stars might help to understand the to date unknown physical mechanisms of the coupling of photospheric processes to the circumstellar environment more clearly. In the OBA supergiants the photosphere-wind connection is heavily veiled by the comparatively strong radiation-driven stellar winds, which themselves are initiated in the stellar photosphere:

In the most luminous supergiant stars, the B hypergiants, the radial wind propagation is the dominant dynamical process. Therefore, the stellar wind becomes the carrier and source of the variability. The less luminous Ia/b supergiants display the most dramatic wind variability, which has been attributed to large-scale wind structures rooted in the stellar photosphere and the modulations driven by the stellar rotation.

The Be stars - as the least extreme extension of this sequence in luminosity - might show the basic physical concepts of this variability more clearly. A promising hint in this direction are the recent works on the Be star $\mu$ Cen by Rivinius et al. (1998, and these proc.) who present observational evidence for a possible feeding of mass into the stellar envelope from the constructive interference of multi-periodic NRP amplitudes. This finding lets me at least speculate if this or a similar photospheric mechanism might also trigger the injecting of matter into streak lines, loops or other large scale wind structures in hot supergiant winds.

\section{References}

Baade, D. 1991, in ESO Conf. 36, Rapid Variability of OB-Stars, ed. D. Baade, 21

Baade, D., 1999, in Proc. IAU Coll. 169, eds. B. Wolf, O. Stahl, A.W. Fullerton, 312

Babel, J., Montmerle, T. 1997, ApJ, 485, L29

Burki, G. 1978, A\&A, 65, 357

Conti, P.S. 1972, ApJ, 174, L79

Ebbets, D. 1982, ApJS, 48, 399

Fullerton, A.W., Gies, D.R., Bolton, C.T. 1991, ApJ, 368, L35

Fullerton, A.W., Massa, D.L., Prinja, R.K., et al. 1997, A\&A, 327, 699

Gagné, M., Caillaut, J.-P., Stauffer, J.R., Linsky, J.L. 1997, ApJ, 478, L87

Gies, D.R., Bolton, C.T. 1986, ApJS, 61, 419

van Genderen, A.M., van de Bosch, F.C., Dessing, F., et al. 1992, A\&A, 264, 88

Henrichs, H.F. 1991, in ESO Conf. 36, Rapid Variability of OB-Stars, ed. D. Baade, 199

Israelian, G., Chentsov, E., Musaev, F. 1997, MNRAS, 290, 521

Kaper, L., Henrichs, H.F., Nichols, J.S. 1996 A\&AS, 116, 257

Kaufer, A., Stahl, O., Wolf, B., et al. 1996a, A\&A, 305, 887

Kaufer, A., Stahl, O., Wolf, B., et al. 1996b, A\&A, 315, 599

Kaufer, A., Stahl, O., Wolf, B., et al. 1997, A\&A, 320, 273 
Kaufer, A. 1998, in Reviews in Modern Astronomy 11, 177 Kudritzki, R.-P. 1999, in Proc. IAU Coll. 169, eds. B. Wolf, O. Stahl, A.W. Fullerton, 405

Lamers, H.J.G.L.M., Bastiaanse, M.V., Aerts, C., et al. 1998, A\&A, 335, 605 van Leeuwen, F., van Genderen, A.M., Zegelaar, I. 1998, A\&AS, 128, 117 Massa, D., Prinja, R.K., Fullerton 1995a, ApJ, 452, 842 Massa, D., Fullerton, A.W., Nichols, J.S., et al. 1995b, ApJ, 452, L53 Mihalas, D., Conti, P.S. 1980, ApJ, 235, 515

Owocki, S.P., Cranmer, S.R., Fullerton, A.W. 1995, ApJ, 453, L37

Prinja, R.K. 1988, MNRAS, 231, 21

Prinja, R.K., Massa, D., Fullerton, A.W. 1995, ApJ, 452, L61

Reid, A.H.N., Bolton, C.T., Crowe, R.A., et al. 1993, ApJ, 417, 320

Reid, A.H.N., Howarth, I.D. 1996, A\&A, 311, 616

Rivinius, Th., Stahl, O., Wolf , B. et al. 1997, A\&A, 318, 819

Rivinius Th., Baade D., Stefl S., et al. 1998, in ASP Conf. Ser. Vol. 135, 343 Stahl, O., Wolf, B., Gäng, Th., et al. 1993, A\&A, 274, L29

Stahl, O., Kaufer, A., Rivinius, Th., et al. 1996, A\&A, 312, 539

Sterken, C. 1977, A\&A, 57, 361

Sterken, C. 1983, in The ESO Messenger 33, 10

Sterken, C., Arentoft, T., Duerbeck, H.W., Brogt, E. 1999, A\&A, 349, 532

Townsend, R.H.D. 1997, MNRAS, 284, 839

Walborn, N.R. 1981, ApJ, 243, L37

Walborn, N.R., Nichols, J.S, 1994, ApJ, 425, L29 